\title{
Ventilação mecânica em crianças
}

\author{
Mechanical ventilation in children
}

Fabio Carmona

\begin{abstract}
RESUMO à ventilação mecânica e seu tratamento imediato.

Palavras-chave: Ventilação Mecânica. Pediatria. Insuficiência Respiratória.
\end{abstract}

O presente artigo apresenta ao leitor as principais indicações de ventilação mecânica assistida em crianças e as principais modalidades existentes, além de trazer orientações para o ajuste dos parâmetros iniciais de ventilação de acordo com a indicação e listar as principais complicações relacionadas

\section{Introdução}

A ampla utilização da ventilação assistida fora da sala de cirurgia ocorreu durante a epidemia de poliomielite da década de 50. ${ }^{1,2}$ Desde então, as técnicas e os aparelhos têm se tornado cada vez mais sofisticados e a ventilação assistida, antes um procedimento realizado somente em unidades de terapia intensiva (UTIs), vem sendo aplicada na sala de urgência e até mesmo em domicílio. Médicos em geral, sobretudo emergencistas e intensivistas, devem estar familiarizados com as técnicas de ventilação hospitalar e doméstica para atenderem adequadamente às necessidades dos pacientes com doença aguda ou agudização de doença crônica. Apesar da maioria dos ventiladores parecer complicada a princípio, o entendimento de alguns princípios básicos da fisiologia respiratória e do funcionamento desses aparelhos possibilita a operação dos mesmos de maneira efetiva e segura. Entretanto, faz-se necessária a compreensão de alguns conceitos importantes.

\section{Conceitos-chave em anatomia e fisiologia}

O ciclo respiratório consiste em inspiração e expiração. A inspiração é um processo ativo em que a contração da musculatura respiratória gera uma pressão negativa intratorácica, deslocando o ar para dentro dos pulmões. A expiração, por outro lado, em condições normais, é um processo passivo, onde a força elástica dos pulmões e da caixa torácica força o ar para fora dos pulmões. A quantidade de ar que entra nos pulmões durante a inspiração depende de dois fatores: complacência e resistência.

Complacência (C) é a capacidade elástica do tórax (que é a soma das complacências dos pulmões e da caixa torácica). Ela pode ser definida como a variação do volume pulmonar $(\Delta \mathrm{V})$ gerada por uma variação de pressão $(\Delta \mathrm{P})$ (Equação 1). Quando a complacência diminui, os pulmões são descritos como "duros". Doenças como a síndrome do desconforto respiratório agudo (SDRA), a doença da membrana hialina
Docente da Divisão de Terapia Intensiva Pediátrica, Departamento de Puericultura e Pediatria, Faculdade de Medicina de Ribeirão Preto, Universidade de São Paulo.
Correspondência: Prof. Dr. Fabio Carmona Avenida dos Bandeirantes 3900 14049-900 - Ribeirão Preto - SP. carmona@fmrp.usp.br

Artigo recebido em 11/04/2012 Aprovado para publicação em 20/06/2012 
e o edema pulmonar cursam com diminuição da complacência pulmonar. ${ }^{1}$

$$
\mathrm{C}=\Delta \mathrm{V} / \Delta \mathrm{P}(\text { Equação } 1)
$$

Onde: $\mathrm{C}=$ complacência pulmonar, $\Delta \mathrm{V}=$ variação de volume, $\Delta \mathrm{P}=$ variação de pressão.

Resistência (R) é a dificuldade na passagem do ar pelas vias aéreas, podendo ser definida como a variação de pressão $(\Delta \mathrm{P})$ necessária para produzir determinado fluxo (Q) (Equação 2). Doenças como a asma, a bronquiolite e a doença pulmonar obstrutiva crônica (DPOC) cursam com aumento da resistência das vias aéreas. ${ }^{1}$

$$
\mathrm{R}=\Delta \mathrm{P} / \mathrm{Q}(\text { Equação 2) }
$$

Onde: $\mathrm{R}=$ resistência da via aérea, $\Delta \mathrm{P}=$ variação de pressão, $\mathrm{Q}=$ fluxo de gás.

Constante de tempo (CT) é o tempo necessário para que ocorra equilíbrio de pressões entre a via aérea e os alvéolos. Equivale ao produto da resistência pela complacência (Equação 3). Em um recémnascido normal, seu valor aproximado é de 0,15 segundos, enquanto que no adulto normal é de 0,30 segundos. Em geral, 1 CT equilibra $63 \%$ dos alvéolos, 3 CT equilibram $95 \%$ dos alvéolos e 5 CT equilibram 99\% dos alvéolos. Por isso, em geral, o tempo inspiratório deve ser ajustado para 3 a $5 \mathrm{CT}$, o que equivale a 0,45 segundos em recém-nascidos e 1 segundo em adultos. ${ }^{3}$

$$
\mathrm{CT}=\mathrm{R} \times \mathrm{C}(\text { Equação } 3)
$$

Onde: $\mathrm{CT}=$ constante de tempo, $\mathrm{R}=$ resistência da via aérea, $\mathrm{C}=$ complacência pulmonar.

Trabalho respiratório é o esforço feito pelo paciente para vencer a complacência e a resistência do aparelho respiratório. Assim, patologias que reduzem a complacência ou aumentam a resistência do aparelho respiratório causam aumento do trabalho respiratório, levando ao aumento da frequência respiratória e uso da musculatura acessória. ${ }^{2}$

$\mathrm{O}$ volume de ar que entra e sai dos pulmões a cada ciclo respiratório chama-se volume corrente (VC). ${ }^{2} \mathrm{Em}$ pessoas normais varia de 6 a $8 \mathrm{~mL} / \mathrm{kg}$ de peso. A capacidade residual funcional (CRF) é o volume de ar que permanece dentro dos pulmões ao final da expiração normal. É determinada por duas forças opostas: a força elástica dos pulmões que, no fim da expiração favorece o colapso pulmonar, e a força elástica da caixa torácica que, no fim da expiração favorece a expansão pulmonar. ${ }^{1}$ Volume de fechamento pulmonar (VFP) é o volume pulmonar a partir do qual começa a haver colapso das unidades alveolares. Em adultos, a CRF é maior do que o VFP. Assim, condições que aumentem o VFP ou reduzam a CRF levam a colapso pulmonar e atelectasia. Em crianças, ao contrário dos adultos, a CRF é menor do que o VFP, o que explica a maior propensão destes pacientes à formação de atelectasias. ${ }^{3}$ A pressão positiva expiratória final (PEEP), usada durante a ventilação assistida, aumenta a CRF acima do VFP, auxiliando a reexpansão e prevenindo o colapso pulmonar. ${ }^{1}$ (Figura 1 ).

\section{Objetivos primários da ventilação assistida}

Em geral, o objetivo primário da ventilação assistida é auxiliar o funcionamento do aparelho respiratório. Isso pode ser obtido garantindo-se a oxigenação, a ventilação e a relação ventilação/perfusão. ${ }^{4}$

Oxigenação é a passagem de moléculas de oxigênio $\left(\mathrm{O}_{2}\right)$ do espaço alveolar para o sangue, cruzando a membrana alvéolo-capilar. Os principais determinantes da oxigenação são a fração inspirada de oxigênio $\left(\mathrm{FiO}_{2}\right)$ e a pressão média das vias aéreas $\left(\mathbf{P}_{\mathbf{V A}}\right)$ (Equação 4). ${ }^{5}$

$$
\mathrm{PaO}_{2} \sim \mathrm{FiO}_{2} \times \mathrm{P}_{\mathrm{VA}}(\text { Equação } 4)
$$

Onde: $\mathrm{PaO}_{2}=$ pressão parcial arterial de oxigênio, $\mathrm{FiO}_{2}=$ fração inspirada de oxigênio, $\mathrm{P}_{\mathrm{VA}}=$ pressão média nas vias aéreas.

A redução do conteúdo arterial de oxigênio é chamada de hipoxemia, e a redução do conteúdo tecidual de oxigênio, hipóxia. A hipoxemia é definida como a $\mathrm{PaO}_{2}<60 \mathrm{~mm} \mathrm{Hg}\left(\right.$ ou $\left.\mathrm{SaO}_{2}<90 \%\right){ }^{5}$

Ventilação é a troca de gases entre o espaço alveolar e a atmosfera. $\mathrm{O}$ volume de ar que entra e sai dos pulmões em um minuto (volume-minuto) é o principal determinante para a eliminação do dióxido de carbono $\left(\mathrm{CO}_{2}\right)$ do sangue para a atmosfera, uma vez que aquele é extremamente difusível. O volume minuto (Vm) é o produto do $\mathrm{VC}$ pela frequência respiratória (FR) (Equação 5). ${ }^{6}$ 


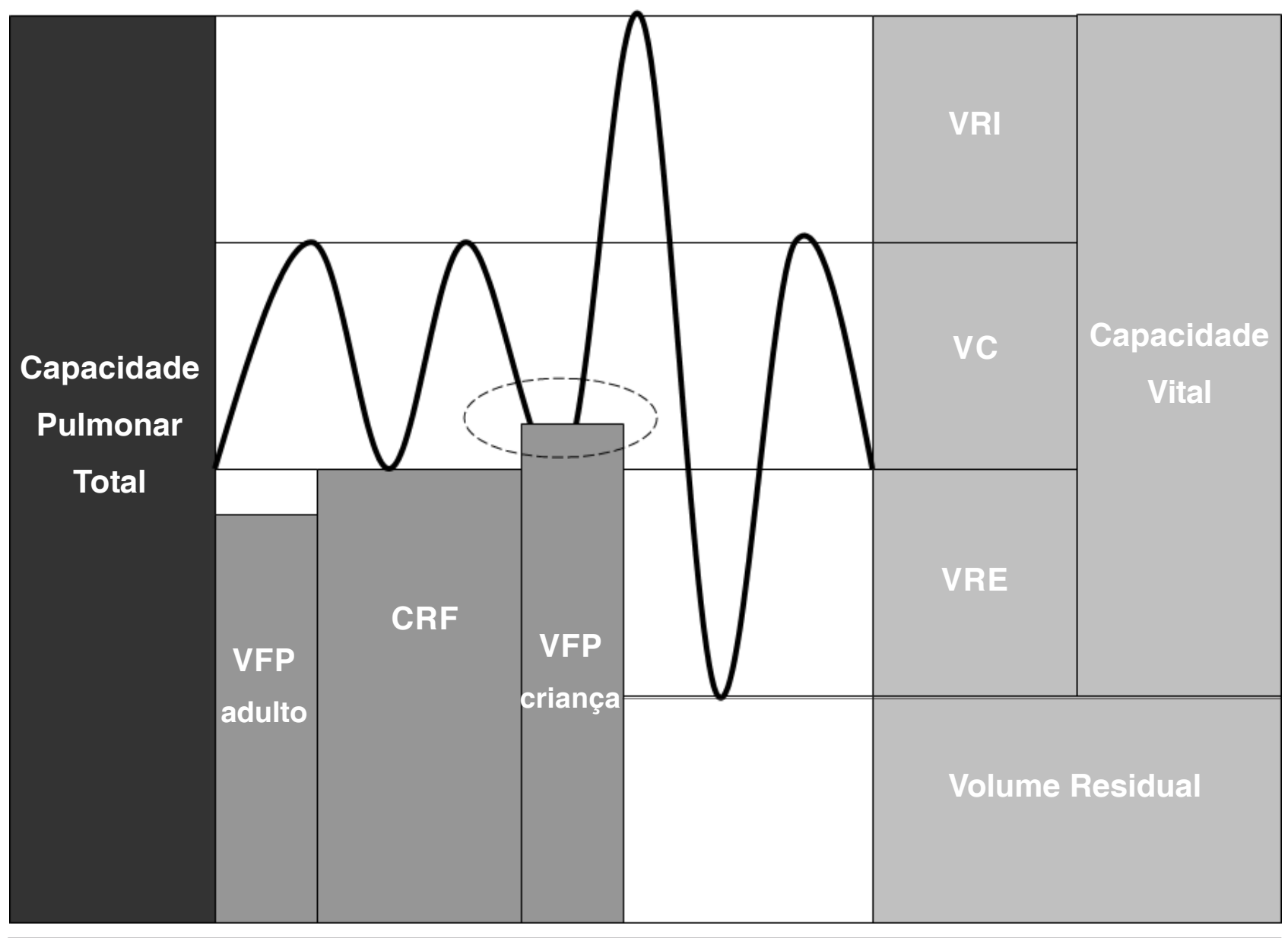

Figura 1. Representação gráfica dos volumes pulmonares durante a respiração normal, inspiração e expiração forçadas. $O$ círculo tracejado mostra como o volume de fechamento pulmonar é atingido durante a respiração normal em crianças. Legenda: VFP= volume de fechamento pulmonar, $\mathrm{CRF}=$ capacidade residual funcional, $\mathrm{VRI}=$ volume de reserva inspiratório, $\mathrm{VC}=$ volume corrente, $\mathrm{VRE}=\mathrm{volume}$ reserva expiratório.

$$
\mathrm{Vm}=\mathrm{VC} \times \mathrm{FR}(\text { Equação 5) }
$$

Onde: $\mathrm{Vm}=$ volume-minuto, $\mathrm{VC}=$ volume corrente, $\mathrm{FR}=$ frequência respiratória.

Dá-se o nome de hipercapnia ao conteúdo aumentado de $\mathrm{CO}_{2}$ no sangue arterial $\left(\mathrm{PaCO}_{2}>45 \mathrm{~mm}\right.$ $\mathrm{Hg}$ ). Acidose respiratória é redução do $\mathrm{pH}$ arterial devido à hipercapnia $(\mathrm{pH}<7,40){ }^{6}$

Relação ventilação/perfusão (V/Q) referese ao fato de que, para haver trocas gasosas, é necessário haver ventilação e perfusão simultaneamente nas mesmas áreas dos pulmões. Há dois tipos de desequilíbrio da relação V/Q: shunt e espaço-morto, ambos cursando com hipoxemia. ${ }^{2}$ Shunt ocorre quanto há áreas pulmonares perfundidas, porém não ventiladas (pneumonia, atelectasia). Assim, o sangue não oxigenado ganha a circulação sistêmica (shunt direita-es- querda). ${ }^{1}$ Espaço-morto ocorre quando há áreas pulmonares ventiladas, porém não perfundidas (embolia pulmonar, hipertensão pulmonar). Assim, o ar retorna à atmosfera sem participar de trocas gasosas. ${ }^{1}$

Falência respiratória é a incapacidade de manter oxigenação e/ou ventilação adequadas, a despeito da utilização de terapias conservadoras (p. ex., administração de oxigênio, broncodilatadores, etc.). ${ }^{4}$ Pode ocorrer na presença de hipoxemia grave $\left(\mathrm{PaO}_{2}<\right.$ $60 \mathrm{~mm} \mathrm{Hg}$ com $\mathrm{FiO}_{2}>60 \%$ ), acidose respiratória grave $\left(\mathrm{PaCO}_{2}>50 \mathrm{~mm} \mathrm{Hg}\right.$ e $\left.\mathrm{pH}<7,25\right)$ ou ambos. As principais causas de falência respiratória são: baixa complacência, alta resistência, desequilíbrio da relação ventilação/perfusão, hipoventilação (central ou periférica), alteração da membrana alvéolo-capilar, trauma crânio-encefálico ou torácico e redução da superfície de troca pulmonar. ${ }^{7}$

Outros objetivos da ventilação assistida incluem: controlar a respiração de maneira específica, para 
o tratamento de doenças extrapulmonares (p. ex., hiperventilação na hipertensão intracraniana, hipoventilação na síndrome da hipoplasia do coração esquerdo com hiperfluxo pulmonar, etc.), reduzir o trabalho respiratório (p. ex., choque séptico ou cardiogênico), ou ainda para administração de medicamentos (óxido nítrico, Heliox®). ${ }^{1}$

\section{Indicações}

A falência respiratória é a indicação primária para o uso da ventilação assistida. Ressalta-se, entretanto, que os valores de $\mathrm{PaO}_{2}, \mathrm{PaCO}_{2}, \mathrm{SaO}_{2}, \mathrm{FiO}_{2}$ e
pH não devem ser usados de maneira absoluta para indicar a ventilação assistida, mas sim analisados em conjunto com a patologia presente, sua provável evolução, o exame físico do paciente e a resposta às demais terapias, além dos riscos associados à ventilação mecânica. ${ }^{5}$ Por outro lado, ao cuidar de um paciente com desconforto respiratório grave, é melhor considerar o início da ventilação assistida sob condições controladas e mais favoráveis do que aguardar a piora da acidose, fadiga e possível falência ou parada cardiorrespiratória. ${ }^{4}$ As principais indicações de ventilação mecânica assistida encontram-se resumidas na Tabela 1.

\section{Tabela 1}

Principais indicações de ventilação mecânica assistida.

\begin{tabular}{|c|c|}
\hline Situação & Indicações \\
\hline Falência respiratória & $\begin{array}{l}\text { - Aumento do trabalho respiratório } \\
\text { Doenças do parênquima pulmonar } \\
\text { - Síndrome do desconforto respiratório agudo (SDRA) } \\
\text { - Pneumonia } \\
\text { - Doença da membrana hialina } \\
\text { - Atelectasia } \\
\text { - Fibrose pulmonar } \\
>\text { Doenças das vias aéreas } \\
\text { - Asma } \\
\text { - Bronquiolite } \\
\text { - Traqueomalácia ou broncomalácia graves } \\
>\text { Alterações da complacência torácica } \\
\text { - Queimaduras extensas } \\
\text { - Traumatismos } \\
\text { - Ascite volumosa } \\
\text { Redução da capacidade de sustentar o trabalho respiratório } \\
>\text { Doenças neuromusculares } \\
>\text { Distúrbios eletrolíticos } \\
\text { - Hipocalemia } \\
\text { - Hipocalcemia } \\
>\text { Alteração do controle da respiração } \\
>\text { Traumatismo crânio-encefálico } \\
>\text { Anestesia geral ou sedação intensa } \\
>\text { Apneia da prematuridade } \\
\text { Doenças neurológicas graves }\end{array}$ \\
\hline Manejo de doenças extrapulmonares & $\begin{array}{l}\text { - Necessidade de controle da ventilação } \\
>\text { Hipertensão intracraniana } \\
>\text { Cardiopatias congênitas } \\
>\text { Hipertensão pulmonar do recém-nascido } \\
\text { - Estados pós-operatórios } \\
\text { - Redução do trabalho respiratório } \\
>\text { Choque séptico ou cardiogênico } \\
>\text { Insuficiência cardíaca grave }\end{array}$ \\
\hline
\end{tabular}




\section{Equipamentos}

Para utilizar corretamente um ventilador, é importante também entender alguns conceitos básicos a respeito do funcionamento desses aparelhos, bem como das principais modalidades de ventilação existentes. ${ }^{1}$ A Figura 2 apresenta um diagrama básico do funcionamento dos ventiladores.

Quando o ventilador aumenta a pressão na via aérea $\left(\mathrm{P}_{\mathrm{VA}}\right)$, a diferença de pressão $(\Delta \mathrm{P})$ irá gerar um fluxo $(\mathrm{Q})$ proporcional à resistência $(\mathrm{R})$, e irá entregar um volume corrente (VC) proporcional à complacência (C) (Equação 6). ${ }^{1}$

$$
\mathrm{P}_{\mathrm{VA}}=\mathrm{Q} \times \mathrm{R}+(\mathrm{VC} \div \mathrm{C})+\mathrm{PEEP}(\text { Equação 6) }
$$

Onde: $\mathrm{PVA}=$ pressão da via aérea, $\mathrm{Q}=$ fluxo de ar, $\mathrm{R}=$ resistência da via aérea, $\mathrm{VC}=$ volume corrente, $\mathrm{C}=$ complacência pulmonar, $\mathrm{PEEP}=$ pressão positiva expiratória final.
Na ventilação mecânica assistida, o ciclo respiratório será composto de quatro fases distintas: transição da expiração para a inspiração (disparo), inspiração, transição da inspiração para a expiração (ciclagem ou ciclo) e expiração. ${ }^{1}$

Disparo refere-se ao modo como o ventilador inicia uma inspiração. $\mathrm{O}$ disparo pode ser feito por tempo ou pressão. Quando uma inspiração é iniciada porque foi atingido um limite de tempo pré-determinado, dizemos que o ventilador é disparado a tempo. Quando o ventilador detecta uma redução na pressão do circuito causada por um esforço inspiratório do paciente e inicia a inspiração, dizemos que ele é disparado a pressão. ${ }^{1,3,6}$

Uma vez disparada a inspiração, o ventilador deverá fornecer um fluxo de ar para dentro dos pulmões até que um determinado limite pré-estabelecido seja atingido. Isto pode ser obtido gerando-se uma pressão positiva constante no circuito, durante um tempo determinado, que irá gerar um fluxo e deslocar uma

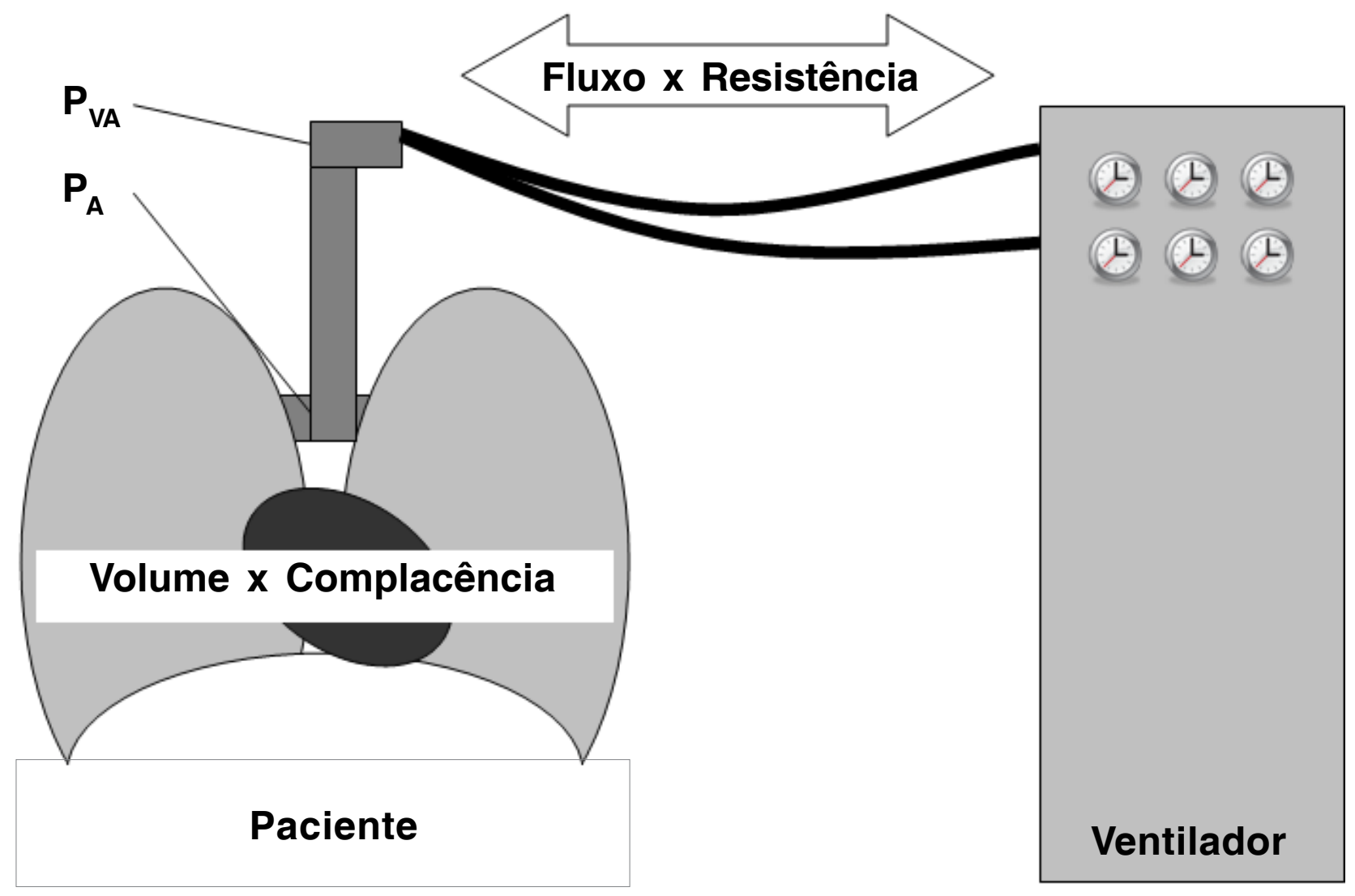

Figura 2. Diagrama básico do funcionamento da ventilação mecânica assistida. Legenda: $P V A=$ pressão na via aérea, $P A=$ pressão alveolar. 
quantidade de ar para dentro dos pulmões. Isto se chama ventilação limitada a pressão. Se o ventilador fornece um volume ou um fluxo pré-determinado, dizemos que a ventilação é limitada a volume ou limitada a fluxo. ${ }^{1,3,6}$

Ciclagem refere-se ao modo como o ventilador encerra a inspiração e passa para a fase expiratória do ciclo. Se a inspiração é encerrada após um tempo pré-determinado, chamamos de ciclagem a tempo. Se a inspiração é encerrada ao atingir-se determinada pressão ou volume, dizemos que se trata de ciclagem a pressão ou ciclagem a volume, respectivamente. ${ }^{1,3,6}$

Por fim, durante a expiração, uma válvula é aberta no circuito do ventilador, deixando o ar sair dos pulmões de maneira passiva, até que um novo disparo ocorra. ${ }^{1,3,6}$

Dessa forma, os ventiladores podem ser classificados de acordo com a forma com que eles operam nas três primeiras fases do ciclo. Um determinado ventilador pode ser classificado como disparado a tempo, limitado a pressão e ciclado a tempo. ${ }^{3}$

A classificação mais utilizada, entretanto, refere-se ao modo de ventilação empregado pelos diferentes ventiladores. Ventiladores modernos são capazes de operar em diferentes modos, e ter diversos mecanismos de disparo, inspiração e ciclagem. Os modos ventilatórios mais comumente utilizados serão descritos a seguir.

\section{Ventilação controlada (CV)}

A ventilação controlada é sempre disparada a tempo. Cada ciclo é iniciado a intervalos regulares prédeterminados de tempo. $\mathrm{O}$ ventilador não responde aos esforços respiratórios do paciente. Por isso, este modo é reservado aos pacientes em apneia, muitas vezes inconscientes ou sob anestesia geral. ${ }^{1,3,5,6}$

\section{Ventilação assistida (AV)}

$\mathrm{Na}$ ventilação assistida, o disparo pode ser a pressão ou a fluxo. Os ciclos respiratórios são iniciados somente pelo esforço inspiratório do paciente, detectado pela redução da pressão ou pelo aumento do fluxo no circuito. Este modo permite ao paciente interagir com o ventilador e controlar sua frequência respiratória de maneira sincronizada. Não deve ser usada em pacientes com apneia. ${ }^{1,3,5,6}$

\section{Ventilação assistido-controlada (A/CV)}

Este modo é uma combinação dos dois anteriores, onde os ciclos respiratórios podem ser disparados pelo esforço do paciente (ciclos assistidos) e, caso este apresente um período de apneia e um intervalo de tempo pré-determinado seja atingido, o ventilador também irá disparar (ciclos controlados). Este modo é especialmente útil em pacientes com variação do nível de consciência ou da força muscular, como por exemplo, pacientes recuperando-se de anestesia geral. ${ }^{1,3,5,6}$

\section{Ventilação mandatória intermitente (IMV)}

Este modo de ventilação assemelha-se ao modo ventilação controlada, exceto pelo fato de que o paciente consegue respirar espontaneamente nos intervalos entre os ciclos controlados. É, portanto, disparado a tempo. Para que o paciente possa respirar entre os ciclos, o ventilador pode gerar um fluxo contínuo de gás no circuito, ou um fluxo intermitente liberado pela abertura de uma válvula em resposta ao esforço do paciente. ${ }^{1,3,5,6}$

\section{Ventilação mandatória intermitente sincroni- zada (SIMV)}

A SIMV é similar à IMV, exceto pelo fato de que os ciclos controlados são disparados de maneira sincronizada aos esforços respiratórios do paciente (disparo a pressão ou fluxo). Caso o paciente não apresente esforço respiratório após um intervalo de tempo pré-estabelecido o ventilador também irá disparar um ciclo (disparo a tempo). O paciente pode respirar espontaneamente entre os ciclos. A vantagem deste modo é a maior interação com o ventilador, proporcionando maior conforto para o paciente. É bastante similar ao modo A/CV. ${ }^{1,3,5,6}$

\section{Ventilação com pressão de suporte (PSV)}

A PSV consiste em ciclos respiratórios disparados a pressão, limitados a pressão e ciclados a fluxo. Quando o paciente apresenta um esforço respiratório o aparelho detecta e fornece uma pressão constante no circuito até que o fluxo de ar para o paciente caia a uma fração do fluxo inicial, que marca o fim da inspiração. Este modo permite ajustar a quantidade de suporte que o paciente precisa. Além disso, a ciclagem a fluxo permite que o paciente controle o tempo inspiratório e o volume corrente. Este modo pode ser associado ao SIMV. ${ }^{1,3,5,6}$

\section{Pressão positiva contínua nas vias aéreas (CPAP)}

Este modo refere-se à aplicação de uma pressão constante na via aérea, objetivando aumentar a CRF, mantendo as vias aéreas abertas, abrindo unida- 
des alveolares colapsadas e prevenindo atelectasias. Isso resulta em complacência mais alta, melhor relação ventilação/perfusão e menor trabalho respiratório. A CPAP pode ser aplicada de maneira não-invasiva, por meio de prongas nasais (em recém-nascidos ou lactentes) ou, menos frequentemente, máscaras nasais ou faciais (crianças maiores, adolescentes e adultos). A CPAP aplicada em cânulas traqueais não é recomendada, pois o circuito gera resistência e espaço morto muito grandes. ${ }^{1,3,5,6,8}$

\section{Pressão positiva contínua nas vias aéreas em dois níveis (BiPAP)}

Este modo combina a aplicação de CPAP com PSV de maneira não-invasiva, por meio de máscaras nasais ou faciais. Em geral, é utilizado em crianças maiores, adolescentes e adultos. , $^{1,5,6}$

\section{Ajustes iniciais do ventilador}

Antes do início da ventilação assistida, deve ser realizada a obtenção de uma via aérea avançada. A intubação orotraqueal é o método mais utilizado. É mandatório confirmar a posição correta do tubo traqueal, por ausculta, radiografia de tórax e determinação do $\mathrm{CO}_{2}$ exalado. O diâmetro interno do tubo traqueal pode ser estimado dividindo-se a idade em anos por quatro, e depois somando-se quatro. A posição de fixação do tubo traqueal ao nível do lábio superior pode ser estimada multiplicando-se o diâmetro do tubo traqueal por três. ${ }^{7}$

Depois de assegurar a via aérea, escolher o ventilador apropriado e o modo a ser utilizado, é necessário fazer os ajustes iniciais. É importante salientar que estes ajustes são apenas um ponto de partida. O médico deverá ajustar o modo e os parâmetros do ventilador de maneira individual, de acordo com as características de cada paciente e da condição que levou à necessidade de ventilação assistida. ${ }^{1,3,5,6,9}$

Os ventiladores limitados a volume geralmente são utilizados para ventilar pacientes adultos ou crianças com mais de $10 \mathrm{~kg}$ de peso. Os ventiladores limitados a pressão geralmente são utilizados em recémnascidos e lactentes. Essa regra, entretanto, não é absoluta. Aparelhos modernos são capazes de oferecer diversos modos ventilatórios em todas as faixas etárias. A seguir, descreveremos os passos necessários para ajustar adequadamente um ventilador.

1. Montagem. Em geral, a montagem do equipamento é feita pela equipe de enfermagem ou de fisiote- rapia respiratória. Entretanto, o médico deve estar familiarizado com esses procedimentos, podendo auxiliar a montagem, ou mesmo fazê-lo em situações excepcionais. Verifique, em especial, as conexões do circuito respiratório, a conexão à rede elétrica e às redes de ar comprimido e de oxigênio.

2. Selecionar o modo de ventilação. Os modos disponíveis dependerão do modelo que estiver sendo utilizado. Os modos mais utilizados são SIMV+PSV ou $\mathrm{A} / \mathrm{CV}$.

3. Ajustar a frequência respiratória. A FR deve ser ajustada de acordo com a idade do paciente (Tabela 2). Em geral, pode-se iniciar a ventilação com uma frequência um pouco mais alta e ir reduzindo à medida que a capacidade respiratória do paciente for melhorando, de forma que haja mais ciclos assistidos do que controlados. ${ }^{1}$

\section{Ajustar o volume corrente ou a pressão inspi- ratória de pico}

a. Nos modos limitados a volume, deve-se ajustar o VC para 6 a $8 \mathrm{~mL} / \mathrm{kg}$. Assegure-se de que a pressão inspiratória de pico (PIP) não ultrapasse 35 $\mathrm{cm} \mathrm{H}_{2} \mathrm{O}$. VC maiores podem ser necessários em caso de escape de ar ao redor da cânula traqueal ou se o circuito for muito compressível.

b. Nos modos limitados a pressão, deve-se selecionar um valor de PIP que produza expansão torácica adequada e murmúrio vesicular bem audível à ausculta. Em geral este valor é entre 15 e 20 $\mathrm{cm} \mathrm{H}_{2} \mathrm{O}$. Nos ventiladores capazes de medir o volume expirado, certifique-se de que esteja entre 6 e $8 \mathrm{~mL} / \mathrm{kg}$. Em alguns ventiladores, não é possível regular diretamente a PIP, mas sim a pressão motriz, ou pressão controlada (driving pressure ou $\triangle \mathrm{P}$ ), que é a pressão a ser aplicada acima da PEEP. Assim, com uma PEEP de $5 \mathrm{~cm}$ $\mathrm{H}_{2} \mathrm{O}$, aplicar uma $\Delta \mathrm{P}$ de $10 \mathrm{~cm} \mathrm{H}_{2} \mathrm{O}$ irá gerar uma PIP de $15 \mathrm{~cm} \mathrm{H}_{2} \mathrm{O}{ }^{6}$

5. Ajustar o tempo inspiratório. Dependendo do modelo do ventilador, pode ser possível ajustar tanto o tempo inspiratório (TI) quanto a relação inspiração:expiração (I:E) (Tabela 2).

a. O TI pode ser calculado da seguinte forma. Dividindo-se 60 segundos pela FR obtemos a duração de cada ciclo. Para manter uma relação I:E de 1:2, o TI deve corresponder a um terço da duração do ciclo. Certifique-se de que o TI corresponda a pelo menos $3 \mathrm{CT}$, caso contrário pode ser necessário utilizar uma FR mais baixa. 
Tabela 2

Ajustes iniciais sugeridos para ventilação mecânica, de acordo com a faixa etária.

\begin{tabular}{lccccc}
\hline Faixa etária & PIP & VC & PEEP & FR & TI \\
\hline Recém-nascidos (até 1 mês) & $15-20$ & $6-8$ & 5 & $30-40$ & $0,4-0,6$ \\
Lactentes (até 2 anos) & $15-20$ & $6-8$ & 5 & $20-30$ & $0,5-0,7$ \\
Pré-escolares (até 6 anos) & $15-20$ & $6-8$ & 5 & $15-25$ & $0,7-0,9$ \\
Escolares (até 10 anos) & $15-20$ & $6-8$ & 5 & $12-20$ & $0,8-1,0$ \\
Adolescentes (até 21 anos) & $15-25$ & $6-8$ & 5 & $10-15$ & $1,0-1,3$ \\
Adultos & $20-25$ & $6-10$ & 5 & $8-10$ & $1,2-1,5$ \\
\hline
\end{tabular}

Legenda: $\mathrm{PIP}=$ pressão inspiratória de pico $\left(\mathrm{cm} \mathrm{H}_{2} \mathrm{O}\right) ; \mathrm{VC}=$ volume corrente $(\mathrm{mL} / \mathrm{kg}) ; \mathrm{PEEP}=$ pressão positiva expiratória final $\left(\mathrm{cm} \mathrm{H}_{2} \mathrm{O}\right)$; $\mathrm{FR}=$ frequência respiratória (ipm); $\mathrm{Tl}=$ tempo inspiratório (s).

b. Quando for possível ajustar a relação I:E, certifique-se de que seja de $1: 2$ ou menor $(1: 3$ ou $1: 4)$, certificando-se de que o TI corresponda a pelo menos $3 \mathrm{CT}$; caso contrário pode ser necessário utilizar uma FR mais baixa ou uma relação I:E maior (no máximo 1:2). ${ }^{5}$

6. Ajustar a pressão de suporte. Se o modo selecionado for SIMV+PSV ou somente PSV, deve-se ajustar o seu valor inicial para $10 \mathrm{~cm} \mathrm{H}_{2} \mathrm{O}$, ou o mesmo valor da $\Delta \mathrm{P}$.

7. Ajustar a sensibilidade. Nos modos em que o disparo pode ser iniciado pelo paciente, é importante ajustar a sensibilidade do aparelho. Isso significa determinar que a intensidade de esforço detectado pelo ventilador será interpretada como esforço inspiratório do paciente. $\mathrm{O}$ esforço pode ser detectado através de variações na pressão da via aérea ou no fluxo do circuito. Em geral, uma sensibilidade entre 2 e $3 \mathrm{~cm} \mathrm{H_{2 }} \mathrm{O}$ ou 2 a $4 \mathrm{~L} / \mathrm{min}$ é adequada.

8. Ajustar a concentração de oxigênio. $\mathrm{A} \mathrm{FiO}_{2}$ deve ser ajustada, inicialmente, para $100 \%$. Depois pode ser reduzida, objetivando-se manter a $\mathrm{SaO}_{2} \geq 90 \%$ com $\mathrm{FiO}_{2} \leq 60 \%$. $^{1}$

9. Ajustar a pressão positiva expiratória final. A PEEP deve ser inicialmente ajustada para $5 \mathrm{~cm}$ $\mathrm{H}_{2} \mathrm{O}$. Este valor deve ser ajustado de acordo com a patologia de base, buscando-se determinar a PEEP ideal, onde análise da curva pressão $\times$ volume pode ser útil. ${ }^{1}$

10. Testar o ventilador. Após o ajuste dos parâmetros da ventilação, o funcionamento do aparelho deve ser testado, conectando-se o circuito a um balão de testes ("pulmão artificial") ou a uma luva estéril, selando bem o escape de ar, para verificar o disparo, a inspiração, a ciclagem e a expiração.

11. Obter o consentimento. Caso o paciente esteja consciente, é indispensável obter seu consentimento antes de conectá-lo ao ventilador, informandoo do que irá ser feito, dos desconfortos que poderá sentir, dos riscos e dos benefícios esperados. Esse procedimento poderá ser reduzido ou omitido em situações de urgência e emergência. ${ }^{4}$

12. Conectar o ventilador ao paciente. Antes de conectar o ventilador, o paciente deverá estar sendo ventilado com dispositivo do tipo bolsa-valva e oxigênio a $100 \%$. Realize uma aspiração da via aérea com técnica estéril. Desconecte a bolsa-valva e conecte o circuito do ventilador à cânula traqueal do paciente. ${ }^{6}$

13. Examinar o paciente. Imediatamente após conectar o ventilador ao paciente, deve-se examinar o paciente com ênfase nos aparelhos respiratório e cardiovascular. Verifique o nível de conforto do paciente, o grau de expansão torácica, a coloração das mucosas e dos leitos ungueais, a presença de escape de ar ao redor da cânula traqueal, a sincronia entre o ventilador e o paciente, ausculte o tórax e certifique-se de haver murmúrio vesicular bilateral. Faça também ausculta cardíaca, palpe os pulsos centrais e periféricos e verifique a frequência cardíaca, a pressão arterial e o tempo de enchimento capilar. O uso de ventilação com pressão positiva pode levar a deterioração cardiovascular pela redução do retorno venoso ao átrio direito e aumento da pós-carga do ventrículo direito. Podem ser necessárias expansões de volume com solução isotônica em alíquotas de 10 a $20 \mathrm{~mL} / \mathrm{kg}^{7}$

14. Verificar o funcionamento do ventilador. Verifique se após a conexão com o paciente o ventilador está operando corretamente e com os parâmetros ajustados inicialmente por você. Faça os ajustes necessários. 
15. Ajustar os alarmes. Os modelos mais modernos de ventilador são equipados com alarmes que detectam mudanças nas pressões das vias aéreas, no volume inspirado ou expirado, na frequência respiratória, na complacência pulmonar, entre outros. Esses alarmes devem estar sempre ligados e regulados de acordo com limites de tolerância prédefinidos pela equipe médica.

16. Monitorização. O paciente em ventilação mecânica deve estar com monitor cardíaco, pulsoxímetro e monitorização não-invasiva da pressão arterial. Adicionalmente, pode-se acrescentar medida do $\mathrm{CO}_{2}$ exalado $\left(\mathrm{ETCO}_{2}\right)$ por um capnógrafo, medida invasiva da pressão arterial e/ou da pressão venosa central (PVC), entre outros. O uso da capnografia pode reduzir o número de coleta de gasometrias arteriais. Deve-se também coletar gasometria arterial e solicitar uma radiografia do tórax. Alguns modelos de ventilador oferecem monitorização da dinâmica ventilatória em tempo real.

17. Ajuste adicionais. Os parâmetros da ventilação devem ser ajustados constantemente para se adequar às necessidades do paciente, de acordo com o exame clínico, a monitorização e a análise da radiografia de tórax e da gasometria arterial.

\section{Sedação e analgesia}

O desconforto causado pela presença da cânula traqueal, procedimentos dolorosos, a pressão positiva intratorácica, associados à ansiedade e a características da patologia de base geralmente tornam necessária a sedação do paciente. Além disso, a sedação pode reduzir o trabalho respiratório e o consumo de oxigênio, auxiliando a terapêutica. Em geral, infusão contínua de um benzodiazepínico (midazolam, 0,1 a $0,3 \mathrm{mg} / \mathrm{kg} / \mathrm{h}$ ) associado a um opioide (fentanil, $1 \mathrm{a}$ $3 \mu \mathrm{g} / \mathrm{kg} / \mathrm{h}$ ) é suficiente para a maioria dos pacientes. ${ }^{7}$ A associação com um bloqueador neuromuscular (vecurônio, 1 a $3 \mu \mathrm{g} / \mathrm{kg} / \mathrm{min}$ ) é reservada a casos selecionados.

\section{Complicações agudas}

O paciente sob ventilação mecânica pode apresentar complicações agudas que podem ser fatais, se não houver intervenção imediata. Frente a um paciente sob ventilação mecânica assistida com deterioração súbita, desconecte o ventilador imediatamente e inicie ventilação com bolsa-valva e oxigênio a $100 \%$. Em seguida, verifique as seguintes situações, que po- dem ser lembradas através da regra mnemônica DOPE ${ }^{7}$ :

- (D) Deslocamento da cânula. Verifique a posição da cânula traqueal e ausculte o tórax do paciente. A cânula pode ter se deslocado para fora da laringe, e o paciente está extubado, ou pode ter se deslocado para dentro, e o paciente está com intubação seletiva de um dos brônquios-fonte (em geral o direito). No primeiro caso, remova a cânula, inicie ventilação com bolsa-valva-máscara e realize nova intubação. No segundo caso, tracione cuidadosamente enquanto ausculta o paciente até que se ouça murmúrio vesicular bilateralmente.

- (O) Obstrução da cânula. Verifique se a cânula traqueal não está dobrada ou obstruída por rolhas de secreção espessa. Realize uma aspiração da cânula com técnica estéril. Em raras situações pode ser necessário trocar a cânula por uma de maior calibre.

- (P) Pneumotórax. Verifique se o tórax expande de maneira simétrica e se o murmúrio vesicular pode ser auscultado bilateralmente. Caso haja redução ou abolição em um dos hemitórax, a percussão do tórax poderá auxiliá-lo a diferenciar o pneumotórax de efusão pleural volumosa ou consolidação pulmonar extensa. A drenagem torácica é o tratamento de escolha. Entretanto, caso o paciente esteja instável ou seu estado se deteriorando, pode-se fazer uma punção de prova com agulha de grosso calibre em selo d'água (na seringa), na face anterior do hemitórax comprometido, na linha hemiclavicular, segundo espaço intercostal (borda superior da costela inferior). Caso haja saída de ar, aspire o máximo possível e depois abra o sistema para o ambiente. Isso transformará o pneumotórax fechado (e hipertensivo) em pneumotórax aberto, e com isso a ventilação com bolsa-valva pode promover a reexpansão pulmonar. A drenagem torácica deve ser realizada o mais rápido possível.

- (E) Falha do equipamento. Verifique novamente o funcionamento do ventilador, os ajustes, a conexão com as fontes de ar comprimido e de oxigênio, a conexão à rede elétrica, a presença de conexões defeituosas, vazamentos de ar, etc. Se tudo estiver aparentemente funcionando e o paciente não tolerar ser reconectado, o ventilador deverá ser substituído por outro.

Pode ainda haver comprometimento hemodinâmico e baixo débito cardíaco por causa da pressão positiva intratorácica. Alguns pacientes podem se be- 
neficiar de expansões de volume intravascular ou de drogas inotrópicas, como a milrinona ou a dopamina, em doses baixas. ${ }^{7}$

\section{Situações especiais}

\section{Doença parenquimatosa pulmonar}

Em pacientes com doenças graves do parênquima pulmonar, em que a complacência está muito reduzida, a redução da CRF pode levar ao colapso de grande parte das unidades alveolares, levando a hipoxemia refratária. Para restaurar a relação ventilação/ perfusão e expandir as unidades alveolares colapsadas deve-se utilizar a PEEP em níveis mais elevados. De maneira geral, a PEEP deve ser aumentada em $2 \mathrm{~cm}$ $\mathrm{H}_{2} \mathrm{O}$ a cada 3 a 5 minutos, até que a oxigenação melhore e seja possível manter a $\mathrm{SaO}_{2} \geq 90 \%$ com $\mathrm{FiO}_{2}$ $\leq 60 \%$. Lembre-se de que a PEEP pode reduzir o retorno venoso direito e aumentar a pós-carga do ventrículo direito, levando a baixo débito cardíaco. Podem ser necessárias novas adequações da volemia com expansões de solução isotônica em alíquotas de 10 a $20 \mathrm{~mL} / \mathrm{kg}^{1,6}$

\section{Obstrução das vias aéreas inferiores}

Doenças que cursam com obstrução das vias aéreas inferiores, como a asma e a bronquiolite, que levam ao aumento acentuado da resistência das vias aéreas, cursam também com hiperinsuflação pulmonar, que pode ser agravada pela pressão positiva. A hiperinsuflação das unidades alveolares, que também são mal-ventiladas e, portanto, hipóxicas, leva a compressão dos capilares pulmonares e aumento da resistência vascular pulmonar. Com isso, há aumento da pós-carga do ventrículo direito e redução do retorno venoso para o átrio esquerdo, contribuindo para baixo débito cardíaco sistêmico. Para evitar esses efeitos, devem-se usar os mínimos valores possíveis de PIP ou VC e PEEP. Além disso, o tempo necessário para completar a expiração pode ser muito maior do que o habitual, devido ao aumento da resistência da via aérea. Por isso, uma nova inspiração pode ser iniciada antes que a expiração termine e atinja a capacidade residual funcional, levando a um "empilhamento" de ciclos respiratórios. Esse fenômeno, conhecido como PEEP inadvertida ou auto-PEEP, agrava a hiperinsuflação e aumenta a pressão média das vias aéreas. Para evitar isso, deve-se reduzir a frequência respiratória ao mínimo possível (mais eficaz) e reduzir a rela- ção I:E para 1:3 ou 1:4 (menos eficaz), aumentando assim o tempo expiratório. ${ }^{1,6}$

\section{Ventilação protetora}

Em algumas situações, podemos utilizar estratégias conhecidas como ventilação protetora, que consiste em tolerar alguns parâmetros da gasometria arterial fora dentro de limites mais amplos do que os da normalidade, em especial a $\mathrm{PaCO}_{2}$ e o pH arterial. A ventilação protetora permite a utilização de ajustes mais "conservadores" no ventilador, de modo a evitar complicações relacionadas ao uso de altas pressões e volumes, como por exemplo, pneumotórax e baixo débito cardíaco, e também reduzir a incidência e a gravidade das lesões pulmonares agudas e crônicas induzidas pela ventilação mecânica. ${ }^{1,6}$ De maneira geral, as estratégias de ventilação protetora são divididas em dois grandes grupos:

\section{Hipercapnia permissiva}

Esta estratégia consiste em usar VC abaixo do recomendado para o peso do paciente (cerca de 4 a 5 $\mathrm{mL} / \mathrm{kg}$ ) e/ou PIP de, no máximo, 35 cm $\mathrm{H}_{2} \mathrm{O}$. O menor volume minuto irá levar a hipercapnia, que em geral é bem tolerada, desde que se mantenha a $\mathrm{PaCO}_{2}$ abaixo de 50-55 mm Hg e, principalmente, o pH acima de 7,20 .

\section{Hipoxemia permissiva}

Esta estratégia consiste em usar $\mathrm{FiO}_{2}$ de até $60 \%$, para evitar os efeitos tóxicos do oxigênio em altas concentrações, e limitar a PEEP em até $15 \mathrm{~cm}$ $\mathrm{H}_{2} \mathrm{O}$, tolerando-se uma $\mathrm{SaO}_{2}$ de pelo menos $85 \%$.

\section{Medidas adicionais}

Conforto físico. Deve-se atentar para o posicionamento confortável do paciente no leito, com controle de temperatura ambiente, luminosidade e ruídos. ${ }^{10}$

Ajustar a temperatura do umidificador. A temperatura ideal do umidificador deve estar entre 30 e $34{ }^{\circ} \mathrm{C} .{ }^{2}$

Fisioterapia respiratória e aspiração da cânula traqueal. A fisioterapia respiratória é fundamental para a utilização segura e eficaz da ventilação mecânica. A comunicação e cooperação entre o médico e o fisioterapeuta podem ser extremamente benéficas para o paciente. A aspiração das secreções traqueais deve ser realizada no mínimo a cada 4 horas, ou mais 
frequentemente, se necessário, podendo ser realizada por fisioterapeutas, pela equipe de enfermagem ou pelo próprio médico. Podem ser necessárias doses adicionais de sedativos e/ou analgésicos para o procedimento. Atentar para a técnica estéril. ${ }^{10}$

\section{Conclusão}

Com o entendimento de conceitos básicos da fisiologia respiratória e treinamento adequado, o médico generalista ou pediatra geral pode iniciar a ventilação assistida mecânica em crianças de maneira rápida e segura, sobretudo em situações de urgência e emergência, favorecendo a estabilização do quadro antes da transferência para uma UTI pediátrica ou neonatal.

\section{Casos clínicos}

\section{Caso 1}

Recém-nascido com 10 dias de vida, $3 \mathrm{~kg}$, é trazido à sala de urgência com história de sonolência e recusa das mamadas há 12 horas. Ao exame encontra-se hipoativo, cianótico, com frequência cardíaca de $180 \mathrm{bpm}$, pressão arterial de 50 x $30 \mathrm{~mm} \mathrm{Hg}$, pulsos centrais fracos, tempo de enchimento capilar de 5 segundos, frequência respiratória de 10 ipm, com retrações intercostais, subcostais e de fúrcula, batimento de aleta nasal e movimentos do tipo "gasping". Ausculta pulmonar normal, sopro cardíaco sistólico +4/+6.

\section{Pergunta-se:}

\section{a. Qual é o diagnóstico?}

- Resposta: O diagnóstico é de recém-nascido com choque circulatório, que pode ser séptico ou cardiogênico (cardiopatia congênita), e falência respiratória. Nessa faixa etária, as causas mais comuns são o choque séptico e a presença de uma cardiopatia congênita.

\section{b. Qual deve ser o atendimento inicial?}

- Resposta: O atendimento inicial deste paciente inclui a abertura das vias aéreas (posicionar e aspirar), ventilação com pressão positiva usando dispositivo bolsa-valva-máscara e oxigênio a 100\%, monitorização completa e obtenção de acesso venoso de grosso calibre e fazer expansão com soro fisiológico $0,9 \% 5 \mathrm{~mL} / \mathrm{kg}$ em 15 minutos, até que se consiga uma radiografia de tórax para verificar o tamanho da área cardíaca; se aumentada, sugere choque cardiogênico e a infusão de volume deve ser cuidadosa. Após ventilar o paciente por aproximadamente 1 minuto, se não houver recuperação de ventilação espontânea efetiva, deve-se proceder à intubação. Após a intubação, poderemos selecionar um ventilador disparado a tempo, limitado a pressão e ciclado a tempo, operando em modo IMV. Os parâmetros iniciais serão: PIP $15 \mathrm{~cm} \mathrm{H}_{2} \mathrm{O}$, PEEP $5 \mathrm{~cm} \mathrm{H}_{2} \mathrm{O}$, FR 30-40 ipm, TI 0,5 s (I:E 1:2), $\mathrm{FiO}_{2} 100 \%$. Atentar, neste caso, para o diagnóstico possível de cardiopatia congênita, onde a utilização de oxigênio em altas concentrações pode agravar o quadro pelo fechamento do canal arterial. Portanto, deve-se providenciar o ecocardiograma o mais rápido possível, para esclarecimento diagnóstico.

\section{Caso 2}

Lactente de 6 meses de idade, $8 \mathrm{~kg}$, com história de febre alta $\left(40{ }^{\circ} \mathrm{C}\right)$, tosse produtiva, espirros e coriza há 4 dias, e queda do estado geral e dispneia com piora progressiva há 2 dias. Ao exame, encontrase em regular estado geral, ativo, cianótico, com frequência cardíaca de 130 bpm, pressão arterial de 80 x $50 \mathrm{~mm} \mathrm{Hg}$, pulsos centrais fortes, tempo de enchimento capilar de 2 segundos, frequência respiratória de 60 ipm, com retrações intercostais, subcostais e de fúrcula e batimento de aleta nasal. À ausculta, murmúrio vesicular reduzido e soproso em todo o hemitórax direito.

\section{Pergunta-se:}

\section{a. Qual é o diagnóstico?}

- Resposta: O diagnóstico é de pneumonia bacteriana com falência respiratória. A história de quadro infeccioso das vias aéreas superiores seguido de desconforto respiratório é sugestiva de infecção bacteriana secundária (pneumonia). A ausculta pulmonar é compatível.

\section{b. Qual deve ser o atendimento inicial?}

- Resposta: O atendimento inicial deste paciente inclui abertura das vias aéreas (posicionamento e aspiração) e administração de oxigênio a $100 \%$ (máscara não reinalante). Caso não haja melhora da $\mathrm{SaO}_{2}$, está indicada a intubação e ventilação mecânica. Como o provável mecanismo da hipoxemia é shunt, a pressão positiva intratorácica pode ser extremamente útil. Após a intubação, podemos selecionar um ventilador disparado a tempo e pressão, limitado a pressão e ciclado a tempo e fluxo, operando em modo SIMV. Os ajustes iniciais serão: PIP $15 \mathrm{~cm} \mathrm{H}_{2} \mathrm{O}, \operatorname{PEEP} 5 \mathrm{~cm}$ 
$\mathrm{H}_{2} \mathrm{O}$, FR 20-30 ipm, TI 0,6 s (I:E 1:2), $\mathrm{FiO}_{2}$ $100 \%$. Além disso, a sensibilidade de disparo será de $2 \mathrm{~cm} \mathrm{H}_{2} \mathrm{O}$ e a pressão de suporte de $10 \mathrm{~cm}$ $\mathrm{H}_{2} \mathrm{O}$. A seguir, deve-se aumentar a PEEP gradativamente até obter uma $\mathrm{SaO}_{2} \geq 90 \%$ com $\mathrm{FiO}_{2}$ $\leq 60 \%$. Em geral, uma PEEP entre 7 e $10 \mathrm{~cm}$ $\mathrm{H}_{2} \mathrm{O}$ é suficiente. A PIP deve ser ajustada de modo a oferecer um volume corrente de $5-6 \mathrm{~mL} /$ $\mathrm{kg}(40-50 \mathrm{~mL})$, limitando-se a PIP a $35 \mathrm{~cm} \mathrm{H}_{2} \mathrm{O}$.

\section{Caso 3}

Adolescente de 15 anos, $60 \mathrm{~kg}$, asmático, tem história de crise de dispneia iniciada há 8 horas. Diversos familiares encontram-se gripados. Ao exame encontra-se extremamente ansioso, pálido, cianótico, frequência cardíaca de $120 \mathrm{bpm}$, pressão arterial de 130 x $80 \mathrm{~mm} \mathrm{Hg}$, pulsos centrais fortes, tempo de enchimento capilar de 2 segundos, frequência respiratória de $30 \mathrm{ipm}$, com retrações intercostais, subcostais e de fúrcula, com esforço expiratório. À ausculta, murmúrio vesicular abafado, com raros sibilos expiratórios e tempo expiratório bastante aumentado.

Pergunta-se:

a. Qual é o diagnóstico?

- Resposta: O diagnóstico é de asma aguda gra- ve com falência respiratória. A história clínica e o exame físico sugerem fortemente este diagnóstico. Em pacientes com asma, a ventilação mecânica deve ser evitada ao máximo, pois o manejo é muito difícil.

\section{b. Qual deve ser o atendimento inicial?}

- O tratamento inicial deste paciente inclui abertura das vias aéreas, oferecimento de oxigênio a 100\% (máscara não reinalante), administração imediata de corticosteroides, broncodilatadores inalados ou endovenosos, podendo-se considerar terapias de exceção, como o sulfato de magnésio. Caso não haja melhora da hipoxemia e/ou da hipercapnia, este paciente deverá ser intubado. Podemos escolher um ventilador disparado a tempo e fluxo, limitado a volume e ciclado a volume e fluxo, operando em modo SIMV. Os ajustes iniciais serão: VC 300-360 mL (5-6 mL/kg), PEEP 3$5 \mathrm{~cm} \mathrm{H}_{2} \mathrm{O}$, FR 10-15 ipm, TI 1-1,5 s (I:E 1:3$1: 4), \mathrm{FiO}_{2} 100 \%$, sensibilidade de disparo de $3 \mathrm{~L} /$ min e pressão de suporte de $8 \mathrm{~cm} \mathrm{H}_{2} \mathrm{O}$. Muita atenção para o desenvolvimento de auto-PEEP. Em geral requer uso de bloqueadores neuromusculares. O paciente deve ser extubado o mais rápido possível.

\begin{abstract}
The present article presents to the reader the main indications for mechanical ventilation in children and the most common modalities; it also gives orientation for the initial ventilator settings according to the indication, and lists the main ventilation-related complications and their immediate treatment.
\end{abstract}

Keywords: Ventilation, Mechanical. Pediatrics. Respiratory Failure.

\section{Referências Bibliográficas}

1. Prabhakaran P, Sasser W, Borasino S. Pediatric mechanical ventilation. Minerva Pediatr. 2011;63(5):411-24.

2. Schmidt GA, Hall JB. Management of the ventilated patient. In: Hall JB, Schmidt GA, Wood LDH, editors. Principles of Critical Care. 3rd ed. New York: McGraw-Hill; 2005.

3. Tilelli JA. Ventilator considerations. In: Baren JM, Rothrock SG, Brennan J, Brown L, editors. Pediatric Emergency Medicine. 1st ed. Philadelphia: Elsevier; 2008. p. 1177-83.

4. Carpenter TC, Dobyns EL, Grayck EN, Mourani PM, Stenmark KR. Critical Care. In: Hay Jr. WW, Levin MJ, Sondheimer JM, Deterding RR, editors. Current Diagnosis \& Treatment: Pediatrics. 19th ed. Columbus: McGraw-Hill Professional; 2009. p. 339-74.

5. Danzl D, Vissers R. Tracheal intubation and mechanical venti- lation. In: Tintinalli J, editor. Emergency Medicine: A Comprehensive Study Guide. 6th ed. New York: McGraw-Hill; 2004.

6. Luria JW. Introduction to conventional mechanical ventilation. In: King C, Henretig FM, editors. Textbook of Pediatric Emergency Procedures. 1st ed. Baltimore: Lippincott Williams \& Wilkins; 2008. p. 807-15.

7. American Heart Association. PALS - Pediatric Advanced Life Support - Provider Manual. Portuguese ed. Hazinski MF, editor. Buenos Aires: Waverly Hispanica S.A.; 2002.

8. Gregoretti C, Pelosi P, Chidini G, Bignamini E, Calderini E. Noninvasive ventilation in pediatric intensive care. Minerva Pediatr. 2010;62(5):437-58.

9. Duyndam A, Ista E, Houmes RJ, van Driel B, Reiss I, Tibboel D. Invasive ventilation modes in children: a systematic review and meta-analysis. Crit Care. 2011;15(1):R24.

10. Carlotti APCP. Manual de Rotinas de Terapia Intensiva Pediátrica. 1st ed. São Paulo: Tecmedd; 2005. 